\title{
Modified negative binomial description of the multiplicity distributions in lepton-nucleon scattering
}

\author{
O. G. TCHIKILEV"2 \\ Institute for High Energy Physics \\ 142284, Protvino, Russia
}

\begin{abstract}
It is shown that charged hadron multiplicity distributions in lepton-nucleon scattering are fairly well described by the modified negative binomial distribution in the energy range from $3-4$ to $\simeq 220 \mathrm{GeV}$. The energy behaviour of the parameter $k$ is similar to the dependence observed for $e^{+} e^{-}$annihilation.
\end{abstract}

( to be published in Phys. Lett. B)

\footnotetext{
${ }^{1}$ This work was partially supported by INTAS, contract INTAS-93-3602

${ }^{2}$ E-mail: tchikilov@mx.ihep.su
} 
In recent papers $[1,2,3,4,5]$ negatively charged particle multiplicity distributions in $e^{+} e^{-}$annihilation have been well described by the modified negative binomial distribution (MNBD) with the parameter $\Delta$ practically energy independent and the parameter $k$ approaching the value 7 . The aim of this letter is to show that the MNBD fairly well describes multiplicity distributions in lepton-nucleon interactions with similar behaviour of the parameters.

Let us remind that the MNBD can be defined by the probability generating function

$$
M(x)=\sum_{n} P_{n} x^{n}=\left(\frac{1+\Delta(1-x)}{1+r(1-x)}\right)^{k},
$$

where $P_{n}$ is the probability to produce $n$ particles. For integer $k$, positive $r$ and negative $\Delta$ lying in the interval $-1 \leq \Delta \leq 0$ the MNBD is positive definite and can be computed as a convolution of the Newton binomial (the numerator in (1)) and the negative binomial (the denominator in (1)), these two distributions are also positive definite. The probabilities $P_{n}$ for the MNBD can be computed also using iteration relations given in the paper [4] or the formulae given in the paper [6].

In tables 1 and 2 the results of the MNBD fits with fixed $\Delta=-0.5$ to the published negatively charged particle multiplicity distributions for the reactions $\mu^{ \pm}+p \rightarrow \mu^{ \pm}+X^{+}[7,8,9]$ and $\mu^{+}+D_{2} \rightarrow \mu^{+}+X^{+}[10]$ are given for different intervals of the invariant mass $W$ of the hadronic system $X$ and the momentum transfer squared $Q^{2}$. The parameter $r$ has been replaced in fits by the the mean multiplicity $<n>$ using the constraint

$$
r=\Delta+<n>/ k \text {. }
$$

The data have been selected using two simple criteria: a) the number of measured points $n_{p}$ in the multiplicity distribution exceeds 3 and b) $W>3 \mathrm{GeV}$. For $\mu^{+} D_{2}$ reaction the published uncorrected multiplicity distributions have been used, since the effect of corrections is rather small as can be seen from the comparison of the uncorrected multiplicity distributions with results of the negative binomial fits to the "unfolded" distributions [10,11].

One can see from tables 1 and 2 that the quality of fits is quite qood, the points with big $\chi^{2} / N D F$ values are mainly at small $W$ and/or $Q^{2}$. Big $\chi^{2} / N D F$ at low $W$ can be explained by the rise of the parameter $k$ from $\simeq 1$ to $\simeq 3$ in the wide $W$ intervals, at higher $W$ the relative variation of the parameter $k$ becomes smaller and therefore its influence is not seen. It is necessary to note that for points with big $\chi^{2} / N D F$ acceptable fits are obtained with free $\Delta$ (not shown). The reasonable fits with free $\Delta$ are obtained also in the energy range $W<3 \mathrm{GeV}$, 
where $n_{p}$ is usually below 4 , i.e. the number of degrees of freedom is too small for two-parameter fits.

In the table 3 the results of the MNBD fits to the multiplicity distributions in the reactions $\nu+p \rightarrow \mu^{-}+X^{++}[12,13], \nu+n \rightarrow \mu^{-}+X^{+}[12]$ and $\bar{\nu}+p \rightarrow$ $\mu^{+}+X^{0}[14]$ are given. For these reactions the quality of fits is also quite qood, big $\chi^{2} / N D F$ again are observed mainly for the $W$ intervals near $3-4 \mathrm{GeV}$.

As seen from tables 1 and 2 the $Q^{2}$ dependence of the parameter $k$ for $\mu p$ reactions in different $W$ intervals is weak or even absent. The energy dependence of the parameter $k$ for the studied lepton-hadron processes is compared in fig. 1 with the energy dependence of the parameter $k$ for $e^{+} e^{-}$annihilation, the $k$ values for $e^{+} e^{-}$data were taken from the papers $[4,5]$. For clarity only the part of the values given in tables 1-3 is shown in this figure. The errors of the parameter $k$ for the studied reactions (not given) are of the order $\simeq 1$ and exceed the errors for statistically more significant $e^{+} e^{-}$data. The $k$ values for $\mu p$ reactions follow the dependence observed for $e^{+} e^{-}$annihilation. The $k$ values for $\nu p$ scattering are slightly below the ones for $e^{+} e^{-}$annihilation and approach them from below with the energy increase whereas the $k$ values for $\bar{\nu} p$ scattering are slightly above the $e^{+} e^{-}$data. The $k$ values for $\mu^{+} D_{2}$ scattering are slightly below the $k$ values for $\mu^{+} p$ scattering.

Recently charged particle multiplicity distributions for the reaction $e^{+}+p \rightarrow$ $e^{+}+X$ have been published by the H1 Collaboration [15]. The results of the MNBD fits with the fixed $k=7$, as suggested by the $e^{+} e^{-}$data, are given in the table 4 for the maximum available pseudorapidity interval $1<\eta^{*}<5$. The $\chi^{2} / N D F$ values for MNBD fits are good, but they should be considered just indicative, since the full covariance matrix is not published and therefore the proper treatment of the correlations between measurements of the neighbour multiplicities is not possible. This contrasts presented earlier fits of the muon and (anti)neutrino data obtained mainly using bubble and streamer chambers, where these correlations are small. Nevertheless one can compare these values with the $\chi^{2} / N D F$, obtained for fits using another generalization of the negative binomial, so called Generalized Negative Binomial Distribution (GNBD) $[16,17]$ (see table 4). The $\chi^{2} / N D F$ values for MNBD fits are significantly smaller than for GNBD fits. It is necessary also to note that MNBD fits are one-parameter fits, since only $\Delta$ is free parameter and $r$ has been fixed using the measured mean multiplicity $\left\langle n_{c h}>\right.$, whereas the GNBD fits are two-parameter fits.

One can also estimate the parameter $k$ from the ratio of the dispersion $D=$ $\left(<n^{2}>-<n>^{2}\right)^{1 / 2}$ to the mean multiplicity $<n>$ using formula

$$
\frac{D^{2}}{<n\rangle^{2}}=\frac{1}{k}+\frac{1+2 \Delta}{<n>}
$$


for $\Delta=-0.5$ it gives

$$
k=\frac{<n>^{2}}{D^{2}} .
$$

This formula has been applied to the data given in the papers $[18,19,20]$, where multiplicity distributions are not tabulated. The ratio of the dispersion $D_{c h}$ to the mean multiplicity for charged particle multiplicity distribution has been calculated from the results of the negative binomial fits (parameters $k_{N B}$ and $\langle n\rangle_{N B}$ ) and has been transformed to the ratio for negative charged particle multiplicity distribution. The final formula for the parameter $k$ (called $\kappa$ to be distinguished from $k$ for MNBD fits) is

$$
\kappa=\left(\frac{k_{N B}<n>_{N B}}{k_{N B}+<n>_{N B}}\right)\left(\frac{<n>_{N B}-|q|}{<n>_{N B}}\right)^{2},
$$

where $\mathrm{q}$ is the total charge of the hadronic system $X$ in the studied reactions.

The energy dependence of the parameter $\kappa$ shown in the fig. 2 is similar (if not coincides) with the energy dependence of the parameter $k$ in the fig. 1 . The $\kappa$ values for the $\mathrm{H} 1$ data, shown in the figure were calculated from the values of the second normalized moment $C_{2}=<n^{2}>/<n>^{2}$ given in 15 for the interval $1<\eta^{*}<5$ using the relation $(<n>/ D)^{2}=1 /\left(C_{2}-1\right)$. The $C_{2}$ for the charged particle multiplicity distribution are used, since at the HERA energies the effect of the total charge $q$ of the system $X$ (second factor in the formula 5) can be neglected.

What is the physical meaning of the MNBD parameters $k$ and $\Delta$ ? One can assume that $k$ is the number of sources of particle production at some stage of interaction, each source develops according to some branching process producing intermediate neutral clusters (as proposed in [1, 3, 歫) The constancy of the parameter $\Delta$ indicates that this branching process is pure birth branching process and in this case $-\Delta$ represents the cluster decay probability into charged hadron pair $(1+\Delta$ is the probabilty of cluster decay into pair of neutral hadrons). Following the statistical model proposed by Goulianos 21] it was assumed in [11, 3, 4] that $\Delta$ should be equal -0.5. But this assumption is incorrect, since it predicts wrong ratio of the average neutral and charged pion multiplicities. In the Goulianos model this deficiency was corrected by "asymmetric" assumption that the neutral cluster decays into charged pion pair or transforms into one neutral hadron. The observed neutral/charged ratio is restored in the model with $2 \pi^{0}$ cluster decay mode if $\Delta=-2 / 3$, this value is close to the value $\Delta=-0.76$ used in the MNBD fits of the $e^{+} e^{-}$data. One should note that the value $\Delta=-2 / 3$ is expected if neutral clusters have zero isotopic spin. The fits of the lepton-nucleon

\footnotetext{
${ }^{3}$ It is of interest to note that the parameter $k$ from MNBD fits is rather close to the parameter $c$ of the Levy parametrization for the KNO scaling function $\psi(z)$ 9, 11, 20]. The possible relation between Levy and MNBD parametrizations has been mentioned in 15$]$.
} 
data with $\Delta$ equal to $-2 / 3$ or -0.76 give also acceptable fits but in general worse compared to the $\Delta=-0.5$.

In conclusion, it is shown that the MNBD with fixed parameter $\Delta=-0.5$ quite well describes lepton-nucleon multiplicity data in the wide energy range

from $3-4$ to $\simeq 220 \mathrm{GeV}$. The parameter $k$ for lepton-nucleon interactions has the energy dependence similar to the energy dependence for $e^{+} e^{-}$annihilation. The evidence, given in this paper, for the same asymptotic value $k=7$ for $e^{+} e^{-}$ annihilation and lepton-nucleon scattering has no direct theoretical explanation now. Future experimental and theoretical studies should show whether this coincidence is accidental or reflects some common dynamical mechanism.

\section{Acknowledgements}

I thank S. Söldner-Rembold and W. Wittek for detailed information on multiplicity distributions in the experiment E665. 


\section{References}

[1] P. V. Chliapnikov and O. G. Tchikilev, Phys. Lett. B242 (1990) 275.

[2] N. Suzuki, M. Biyajima and G. Wilk, Phys. Lett. B268 (1992) 447.

[3] P. V. Chliapnikov and O. G. Tchikilev, Phys. Lett. B282 (1992) 471.

[4] P. V. Chliapnikov, O. G. Tchikilev and V. A. Uvarov, Phys. Lett. B352 (1995) 461.

[5] O. G. Tchikilev, Phys. Lett. B382 (1996) 296.

[6] N. Suzuki, M. Biyajima and N. Nakajima, Phys. Rev. D53 (1996) 3582.

[7] J. Ballam et al., Phys. Lett. 56B (1975) 193.

[8] C. del Papa et al., Phys. Rev. D13 (1976) 2934.

[9] EMC Collab., M. Arneodo et al., Nucl. Phys. B258 (1985) 249.

[10] E665 Collab., M. Adams et al., Z. Phys. C61 (1994) 179.

[11] S. Söldner-Rembold, "Die erzeugung von hadronen in der myon-streuung an Deuterium- und Xenonkernen bei $480 \mathrm{GeV}$ ", PhD thesis, preprint MPIPhE/92-17, 1992.

[12] D. Zieminska et al., Phys. Rev. D27 (1983) 47.

[13] J. Chapman et al., Phys. Rev. Lett. 36 (1976) 124.

[14] M. Derrick et al., Phys. Rev. D25 (1982) 624.

[15] H1 Collab., S. Aid et al., "Charged particle multiplicities in deep inelastic scattering at HERA", preprint DESY 96-160 and hep-ex/9608011, 1996.

[16] S. Hegyi, "Multiplicity distributions in strong interactions:

a generalized negative binomial model", preprint hep-ph/9608346, 1996.

[17] S. Hegyi, "Testing QCD predictions for multiplicity distributions at HERA", preprint hep-ph/9608479, 1996.

[18] EMC Collab., M. Arneodo et al., Z. Phys. C35 (1987) 335.

[19] WA25 Collab., B. Jongejans et al., Nuovo Cimento 101A (1989) 435.

[20] WA21 Collab., G. Jones et al., Z. Phys. C54 (1992) 45.

[21] K. Goulianos, Phys. Lett. 193B (1987) 151. 


\section{$\underline{\text { Figure Captions }}$}

Fig.1 The energy dependence of the parameter $k$ obtained from the MNBD fits with fixed $\Delta=-0.5$ to the negative charged particle multiplicity distributions in the lepton-nucleon interactions compared to the $k$ values for $e^{+} e^{-}$annihilation. For clarity some points are slightly shifted from integer values. The histograms are drawn to guide the eye.

Fig.2 The energy dependence of the parameter $\kappa=(<n>/ D)^{2}=1 /\left(C_{2}-1\right)$ for the reactions $e^{+}+p \rightarrow e^{+}+X$ [15], $\mu^{+}+p \rightarrow \mu^{+}+X$ [18], $\bar{\nu}+p \rightarrow \mu^{+}+X$ [19, 20] and $\nu+p \rightarrow \mu^{-}+X$ [19, 20] compared to the $k$ values for $e^{+} e^{-}$annihilation. For the $\mathrm{H} 1$ data the measurements in the pseudorapidity interval $1<\eta^{*}<5$ are used. 
Table 1: Results of the modified negative binomial fits with fixed parameter $\Delta=-0.5$ to the negative charged particle multiplicity distributions in lepton-hadron reactions $\mu^{+}+p \rightarrow \mu^{+}+X[9], \mu^{-}+p \rightarrow \mu^{-}+X[7]$ and $\mu^{+}+D_{2} \rightarrow \mu^{+}+X[10]$.

\begin{tabular}{|c|c|c|c|c|c|}
\hline Experiment & $\overline{\mathrm{W}(\mathrm{GeV})}$ & 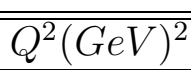 & $\left\langle<n_{-}\right\rangle$(fit) & $\overline{\mathrm{k}}$ & $\overline{\chi^{2} / \mathrm{NDF}}$ \\
\hline \multirow{8}{*}{$\begin{array}{c}\text { J.Ballam [7] } \\
\mu^{-} p \\
16 \mathrm{GeV} / \mathrm{c}\end{array}$} & \multirow{4}{*}{$2.8 \div 3.8$} & 0.24 & $\bar{~} 1.146 \pm 0.018$ & $\overline{22}$ & "10.9/3 \\
\hline & & 0.54 & $1.017 \pm 0.018$ & 2 & $7.0 / 3$ \\
\hline & & 0.95 & $1.071 \pm 0.024$ & 2 & $7.0 / 3$ \\
\hline & & 1.80 & $0.782 \pm 0.076$ & 1 & $13.1 / 3$ \\
\hline & \multirow{4}{*}{$3.8 \div 5.0$} & 0.0675 & $1.222 \pm 0.029$ & 3 & $30.4 / 3$ \\
\hline & & 0.25 & $1.181 \pm 0.075$ & 2 & $5.8 / 3$ \\
\hline & & 0.56 & $1.354 \pm 0.131$ & 2 & $0.8 / 3$ \\
\hline & & 1.38 & $1.151 \pm 0.056$ & 3 & $0.2 / 3$ \\
\hline \multirow{8}{*}{$\begin{array}{c}\text { NA9 } 9 \\
\mu^{+} p \\
280 \mathrm{GeV} / \mathrm{c}\end{array}$} & $4 \div 6$ & \multirow{8}{*}{$4 \div 200$} & $1.754 \pm 0.071$ & 4 & $1.3 / 5$ \\
\hline & $6 \div 8$ & & $2.067 \pm 0.086$ & 3 & $3.3 / 5$ \\
\hline & $8 \div 10$ & & $2.339 \pm 0.037$ & 4 & $9.4 / 6$ \\
\hline & $10 \div 12$ & & $2.598 \pm 0.080$ & 5 & $19.2 / 6$ \\
\hline & $12 \div 14$ & & $2.848 \pm 0.083$ & 5 & $6.8 / 6$ \\
\hline & $14 \div 16$ & & $3.011 \pm 0.072$ & 6 & $7.2 / 7$ \\
\hline & $16 \div 18$ & & $3.108 \pm 0.081$ & 6 & $19.1 / 8$ \\
\hline & $18 \div 20$ & & $3.206 \pm 0.068$ & 7 & $39.6 / 8$ \\
\hline \multirow{9}{*}{$\begin{array}{c}\mathrm{E} 665 \llbracket 10 \\
\mu^{+} D_{2} \\
490 \mathrm{GeV} / \mathrm{c}\end{array}$} & $8 \div 10$ & \multirow{9}{*}{$>1.0$} & $4.984 \pm 0.108$ & 4 & $4.9 / 14$ \\
\hline & $10 \div-12$ & & $5.474 \pm 0.095$ & 4 & $17.6 / 15$ \\
\hline & $12 \div-14$ & & $5.943 \pm 0.106$ & 4 & $33.3 / 17$ \\
\hline & $14 \div 16$ & & $6.525 \pm 0.115$ & 4 & $21.4 / 19$ \\
\hline & $16 \div 18$ & & $6.787 \pm 0.112$ & 4 & $36.4 / 21$ \\
\hline & $18 \div 20$ & & $7.336 \pm 0.131$ & 4 & $30.4 / 19$ \\
\hline & $20 \div 22$ & & $7.241 \pm 0.131$ & 5 & $22.2 / 21$ \\
\hline & $22 \div 25$ & & $7.813 \pm 0.135$ & 4 & $36.4 / 23$ \\
\hline & $25 \div 30$ & & $7.861 \pm 0.156$ & 4 & $28.3 / 22$ \\
\hline
\end{tabular}


Table 2: The same as in Table 1 for reaction $\mu^{+}+p \rightarrow \mu^{+}+X$ at $14 \mathrm{GeV} / \mathrm{c}$ [8].

\begin{tabular}{|c|c|c|c|c|}
\hline \hline $\mathrm{W}(\mathrm{GeV})$ & $Q^{2}(\mathrm{GeV})^{2}$ & $<n_{-}>(\mathrm{fit})$ & $\mathrm{k}$ & $\chi^{2} / \mathrm{NDF}$ \\
\hline \hline 3.1 & & $1.072 \pm 0.075$ & 2 & $8.0 / 3$ \\
3.5 & & $1.328 \pm 0.135$ & 3 & $3.0 / 3$ \\
3.7 & $0.3 \div 0.5$ & $1.225 \pm 0.117$ & 2 & $2.7 / 3$ \\
3.9 & & $1.325 \pm 0.138$ & 2 & $14.2 / 3$ \\
4.3 & & $1.378 \pm 0.116$ & 4 & $6.8 / 3$ \\
\hline 3.1 & & $1.002 \pm 0.036$ & 2 & $13.8 / 3$ \\
3.3 & & $1.094 \pm 0.070$ & 2 & $0.1 / 3$ \\
3.5 & 0.68 & $1.028 \pm 0.069$ & 2 & $7.2 / 3$ \\
3.7 & & $1.207 \pm 0.055$ & 3 & $0.9 / 3$ \\
3.9 & & $1.203 \pm 0.079$ & 2 & $2.6 / 3$ \\
4.3 & & $1.368 \pm 0.131$ & 2 & $8.6 / 3$ \\
\hline 3.1 & & $1.405 \pm 0.052$ & 2 & $12.4 / 3$ \\
3.3 & & $1.119 \pm 0.024$ & 3 & $1.3 / 3$ \\
3.5 & 1.75 & $1.061 \pm 0.038$ & 2 & $10.0 / 3$ \\
3.7 & & $1.150 \pm 0.075$ & 2 & $6.3 / 3$ \\
3.9 & & $1.141 \pm 0.180$ & 1 & $1.5 / 3$ \\
4.3 & & $1.328 \pm 0.121$ & 2 & $3.7 / 3$ \\
\hline & $0.3 \div 0.5$ & $1.042 \pm 0.037$ & 2 & $25.3 / 3$ \\
& $0.5 \div 1.0$ & $1.002 \pm 0.033$ & 2 & $17.4 / 3$ \\
$2.8 \div 3.6$ & $1.0 \div 1.5$ & $0.987 \pm 0.033$ & 2 & $7.7 / 3$ \\
& $1.5 \div 2.0$ & $1.027 \pm 0.037$ & 2 & $11.3 / 3$ \\
& $2.0 \div 4.5$ & $1.054 \pm 0.038$ & 2 & $1.4 / 3$ \\
\hline & $0.3 \div 0.5$ & $1.296 \pm 0.074$ & 3 & $23.7 / 3$ \\
& $0.5 \div 1.0$ & $1.358 \pm 0.074$ & 2 & $10.2 / 3$ \\
$3.6 \div 4.7$ & $1.0 \div 1.5$ & $1.163 \pm 0.052$ & 2 & $4.5 / 3$ \\
& $1.5 \div 2.0$ & $1.279 \pm 0.105$ & 3 & $1.4 / 3$ \\
& $2.0 \div 4.5$ & $1.261 \pm 0.147$ & 2 & $4.8 / 3$ \\
\hline
\end{tabular}


Table 3: The same as in Table 1 for $\nu p \rightarrow \mu^{-}+X^{++}[12,13], \nu n \rightarrow \mu^{-}+X^{+}[12]$ and $\bar{\nu} p \rightarrow \mu^{+}+X^{0}[14]$ reactions.

\begin{tabular}{|c|c|c|c|c|}
\hline Experiment & $\overline{\mathrm{W}(\mathrm{GeV})}$ & $<n_{-}>$(fit) & $\mathrm{k}$ & $\chi^{2} / \mathrm{NDF}$ \\
\hline & $2.83 \div 3.46$ & $0.766 \pm 0.022$ & 1 & $32.6 / 3$ \\
\hline & $3.46 \div 4.0$ & $1.130 \pm 0.024$ & 2 & $3.0 / 5$ \\
\hline & $4.0 \div 4.8$ & $1.287 \pm 0.030$ & 2 & $21.8 / 5$ \\
\hline$\nu p$ & $4.8 \div 5.66$ & $1.606 \pm 0.036$ & 3 & $5.7 / 5$ \\
\hline \multirow[t]{5}{*}{ D. Zieminska 12 } & $5.66 \div 6.76$ & $1.860 \pm 0.047$ & 3 & $12.4 / 6$ \\
\hline & $6.76 \div 7.94$ & $2.048 \pm 0.068$ & 3 & $13.2 / 5$ \\
\hline & $7.94 \div 9.49$ & $2.356 \pm 0.078$ & 4 & $9.7 / 6$ \\
\hline & $9.49 \div 11.18$ & $2.642 \pm 0.124$ & 4 & $13.1 / 6$ \\
\hline & $11.18 \div 15.0$ & $2.710 \pm 0.151$ & 4 & $3.8 / 6$ \\
\hline \multirow{9}{*}{$\begin{array}{c}\nu n \\
\text { D. Zieminska } 12\end{array}$} & $2.83 \div 3.46$ & $1.036 \pm 0.012$ & 2 & $24.0 / 3$ \\
\hline & $3.46 \div 4.0$ & $1.185 \pm 0.020$ & 2 & $25.2 / 4$ \\
\hline & $4.0 \div 4.8$ & $1.560 \pm 0.020$ & 3 & $13.0 / 5$ \\
\hline & $4.8 \div 5.66$ & $1.721 \pm 0.030$ & 3 & $8.1 / 5$ \\
\hline & $5.66 \div 6.76$ & $1.990 \pm 0.048$ & 3 & $3.7 / 5$ \\
\hline & $6.76 \div 7.94$ & $2.217 \pm 0.054$ & 4 & $1.7 / 5$ \\
\hline & $7.94 \div 9.49$ & $2.476 \pm 0.076$ & 4 & $3.4 / 6$ \\
\hline & $9.49 \div 11.18$ & $2.004 \pm 0.036$ & 5 & $9.8 / 6$ \\
\hline & $11.18 \div 15.0$ & $3.128 \pm 0.187$ & 3 & $5.8 / 6$ \\
\hline \multirow{4}{*}{$\begin{array}{c}\nu p \\
\text { J. Chapman 13 }\end{array}$} & $3.16 \div 4.47$ & $1.065 \pm 0.065$ & 2 & $2.8 / 3$ \\
\hline & $4.47 \div 5.48$ & $1.279 \pm 0.085$ & 2 & $2.8 / 4$ \\
\hline & $5.48 \div 7.07$ & $1.776 \pm 0.102$ & 3 & $15.3 / 4$ \\
\hline & $7.07 \div 10$ & $1.564 \pm 0.130$ & 2 & $9.0 / 6$ \\
\hline \multirow{6}{*}{$\begin{array}{c}\bar{\nu} p \\
\text { M. Derrick }[14]\end{array}$} & $3.0 \div 3.5$ & $1.196 \pm 0.042$ & 2 & $7.5 / 3$ \\
\hline & $3.5 \div 4.0$ & $1.545 \pm 0.033$ & 3 & $9.4 / 3$ \\
\hline & $4.0 \div 4.5$ & $1.864 \pm 0.069$ & 4 & $3.7 / 3$ \\
\hline & $4.5 \div 5.0$ & $1.899 \pm 0.088$ & 4 & $3.4 / 3$ \\
\hline & $5.0 \div 7.5$ & $2.510 \pm 0.073$ & 5 & $13.3 / 4$ \\
\hline & $7.5 \div 10.0$ & $2.840 \pm 0.175$ & 8 & $0.3 / 4$ \\
\hline
\end{tabular}


Table 4: The results of the MNBD fits with $k=7$ to the H1 data [15] with $<n_{c h}>$ fixed at the published values for the pseudorapidity interval $1<\eta^{*}<5$ and for the $Q^{2}$ interval $10<Q^{2}<1000(\mathrm{Gev} / \mathrm{c})^{2}$. In last column the $\chi^{2} / N D F$ for fits using Generalized Negative Binomial Distribution from the paper [17] are shown.

\begin{tabular}{|c|c|c|c|}
\hline \hline $\mathrm{W}(\mathrm{Gev})$ & $-\Delta$ & $\chi^{2} / \mathrm{NDF}$ & $\chi^{2} / \mathrm{NDF}($ from $[17])$ \\
\hline \hline $80 \div 115$ & $0.216 \pm 0.034$ & $2.7 / 18$ & $12 / 17$ \\
$115 \div 150$ & $0.233 \pm 0.036$ & $10.6 / 21$ & $26 / 20$ \\
$150 \div 185$ & $0.187 \pm 0.049$ & $4.6 / 22$ & $19 / 21$ \\
$185 \div 220$ & $0.264 \pm 0.057$ & $11.3 / 23$ & $16 / 22$ \\
\hline
\end{tabular}




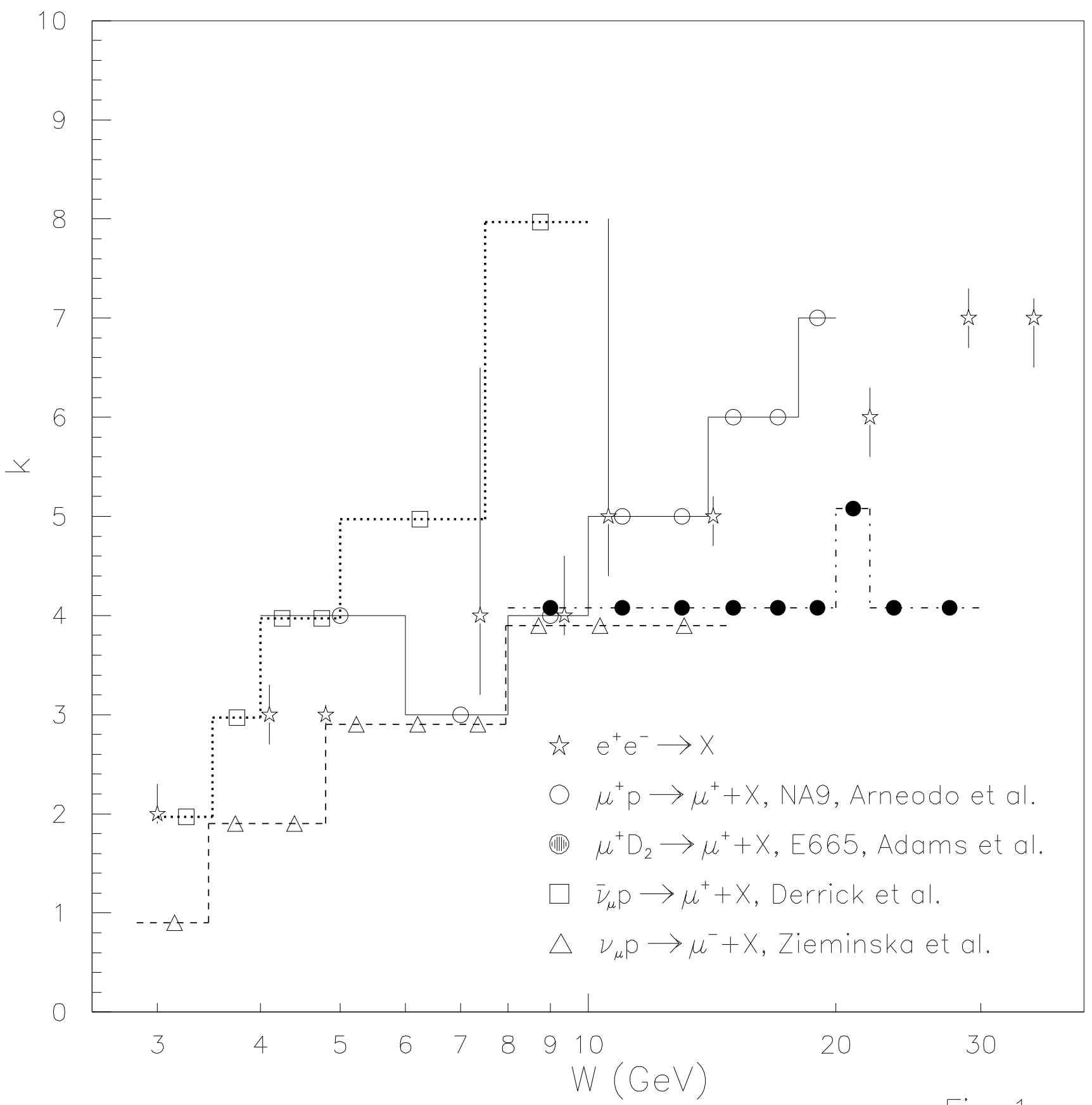

Fig. 1 


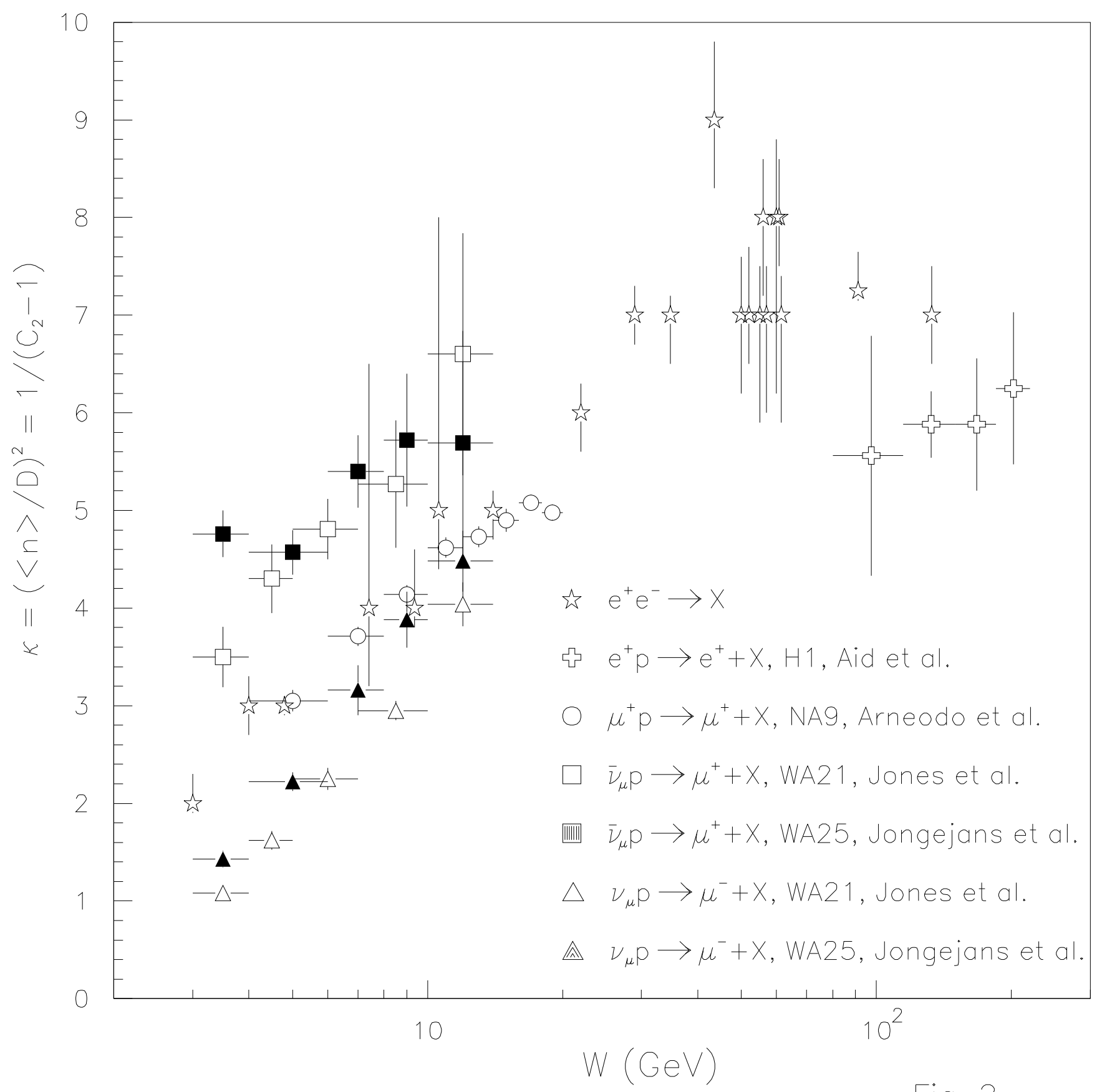

Fig. 2 